\title{
Superconducting Terahertz Sources with 12\% Power Efficiency
}

\author{
R. Cattaneo, ${ }^{1}$ E.A. Borodianskyi, ${ }^{1}$ A.A. Kalenyuk $\odot,{ }^{1,2}$ and V.M. Krasnov ${ }^{1,3,}{ }^{*}$ \\ ${ }^{1}$ Department of Physics, Stockholm University, AlbaNova University Center, Stockholm SE-10691, Sweden \\ ${ }^{2}$ Institute of Metal Physics of National Academy of Sciences of Ukraine, Kyiv 03142, Ukraine \\ ${ }^{3}$ Moscow Institute of Physics and Technology, State University, 9 Institutsiy per., Dolgoprudny 141700, Russia
}

(Received 15 February 2021; revised 30 August 2021; accepted 11 November 2021; published 10 December 2021)

Low power efficiency is one of the main problems of terahertz (THz) sources, colloquially known as "the THz gap." In this work we present prototypes of $\mathrm{THz}$ devices based on whisker crystals of a high-temperature superconductor $\mathrm{Bi}_{2} \mathrm{Sr}_{2} \mathrm{CaCu}_{2} \mathrm{O}_{8+\delta}$ with a record high-radiation power efficiency of $12 \%$ at a frequency of approximately $4 \mathrm{THz}$. We employ various on- and off-chip detection techniques and, in particular, use the radiative cooling phenomenon for accurate evaluation of the emission power. We conclude that such devices can be used for creation of tunable, monochromatic, cw, compact, and powerefficient $\mathrm{THz}$ sources.

DOI: 10.1103/PhysRevApplied.16.L061001

Tunable, monochromatic, cw, compact, and powerefficient sources of terahertz (THz) electromagnetic waves (EMW) are required for a broad variety of applications such as spectroscopy, environmental control, security, nonionizing medical imaging, ultrahigh-speed telecommunication and electronics, as well as for fundamental research in various areas of science [1,2]. The key problem of $\mathrm{THz}$ sources, colloquially known as the "THz gap," is a rapid decay of radiation power efficiency (RPE), i.e., the ratio of emitted and dissipated power [3], in the low $\mathrm{THz}$ range [1]. Despite a significant progress achieved in development of semiconducting quantum cascade lasers (QCLs) [4-6], the RPE of cw QCLs drops from $\simeq 28 \%$ at $f \simeq 55 \mathrm{THz}$ [7] to a subpercent at 3-4 THz [8,9] and to approximately $0.01 \%$ at $f \simeq 1.3 \mathrm{THz}$ [10]. Although QCL frequency can be tuned $[4,5,9,11,12]$, this comes at the expense of a dramatic reduction of RPE to approximately $0.0001 \%$ [11] when low $\mathrm{THz}$ emission is obtained by mixing or downconversion of higher frequencies [11,12]. QCLs emitting at primary low $\mathrm{THz}$ frequencies, on the other hand, have to be cooled down to cryogenic temperatures, $k_{B} T \lesssim h f$ [8-10].

Superconducting devices, based on arrays of Josephson junctions (JJs) have an inherent frequency tunability and provide and alternative technology for creation

\footnotetext{
*Vladimir.Krasnov@fysik.su.se
}

Published by the American Physical Society under the terms of the Creative Commons Attribution 4.0 International license. Further distribution of this work must maintain attribution to the author(s) and the published article's title, journal citation, and DOI. Funded by Bibsam. of cryogenic $\mathrm{THz}$ sources with tunable, monochromatic $\mathrm{cw}$ operation [13-30]. The Josephson frequency, $f_{J}=$ $(2 e / h) V$, is proportional to the dc-bias voltage $V$ and is limited only by the superconducting energy gap, which can be in excess of $30 \mathrm{THz}$ for high- $T_{c}$ superconductors $[31,32]$. Emission with a sub-mW power at $f \simeq 0.5 \mathrm{THz}$ was achieved from large-area mesa structures etched on top of single crystals of a layered cuprate superconductor $\mathrm{Bi}_{2} \mathrm{Sr}_{2} \mathrm{CaCu}_{2} \mathrm{O}_{8+\delta}$ (Bi-2212) [17]. Such mesas represent stacks of atomic scale intrinsic JJs [33]. Tunable EMW emission at the primary Josephson frequency in the whole $\mathrm{THz}$ range 1-11 $\mathrm{THz}$ has been reported from small-area Bi-2212 mesas [21], albeit with a lower power. The RPE for both large [17] and small [21] mesas on Bi-2212 single crystals is $\lesssim 1 \%$, which is decent for $\mathrm{THz}$ sources, but small compared to the theoretical limit of $50 \%$ [34]. The suboptimal operation is caused by impedance mismatching between a device and open space. Another key limitation for all cryogenic devices is set by self-heating. Taking into account limited cooling power of compact cryorefrigerators (sub-Watt at low T), devices with RPE 1\% would not be able to emit much more than $1 \mathrm{~mW}$. Therefore, further enhancement of the emission power from compact cryogenic devices may only be achieved via enhancement of RPE.

Here we present prototypes of $\mathrm{THz}$ sources based on Bi-2212 whisker-type crystals with intermediate-size mesa structures. We employ various techniques for detection of $\mathrm{THz}$ radiation such as in situ detection by a mesa on the same whisker, on-chip detection by electrically isolated mesa, and off-chip detection by a bolometer. Furthermore, we employ the radiative cooling phenomenon for estimation of the absolute value of emitted power. It reveals that 
the RPE of our devices can reach $12 \%$ making a significant step forward towards the theoretical limit of $50 \%$. The boost of efficiency is attributed to good impedance matching with open space, caused by a specific turnstile antennalike geometry of our devices. We conclude that such devices can be used for creation of tunable, and, what is more, power-efficient $\mathrm{cw} \mathrm{THz} \mathrm{sources.}$

Figures 1(a) and 1(b) show an image and a sketch of studied devices. In the middle of each device there is a Bi-2212 whisker with typical sizes $(300-500) \times(20-$ $30) \times(1-5) \mu \mathrm{m}^{3}$ along crystallographic $a, b$, and $c$ axes, respectively. Several metallic electrodes with the width $10-15 \mu \mathrm{m}$ are made across the whisker. Beneath each electrode there is a mesa structure containing $N \sim 150-250$ intrinsic JJs. Devices are made by conventional microfabrication techniques. Details about sample fabrication and the experimental setup can be found in Refs. [21,32] and the Supplemental Material [35].

Figure 1(c) shows the current-voltage $(I-V)$ characteristics of a mesa at $T \simeq 3 \mathrm{~K}$. It consists of multiple branches, due to one-by-one switching of JJs from the superconducting to the resistive state [31-33]. The total number of JJs, $N \simeq 150 \pm 10$ for this mesa, is obtained by counting branches. A rough estimation, valid for all our mesas is $N \simeq V_{\max } / 10 \mathrm{mV}$, where $V_{\max }$ is the voltage at a maximum in the $I-V$. The maximum is caused by back bending at high bias due to self-heating. The extent of self-heating depends on geometry and decreases with decreasing mesa sizes $[36,37]$. Sizes of our mesas are in the range $(2.5-10) \times$ $(10-30) \mu \mathrm{m}^{2}$ with areas $50<A<300 \mu \mathrm{m}^{2}$, which are significantly smaller than "large" mesas $\left(A>10^{4} \mu \mathrm{m}^{2}\right)$ studied in the majority of earlier works [16-20,23-29], but larger than "small" mesas $\left(A \lesssim 10 \mu \mathrm{m}^{2}\right)$ studied in Ref. [21]. Thus, our mesas are of "intermediate size." This enables a significant overall power approximately $1 \mathrm{~mW}$ with a tolerable self-heating.

To analyze EMW emission, we start with an on-chip generation-detection scheme [21] using one mesa as a generator and another mesa on the same chip as a switchingcurrent detector. Figure 1(d) shows evolution of the $I-V \mathrm{~s}$ of the detector at three bias points $\left(I_{\text {gen }}, V_{\text {gen }}\right)$ in the generator. The $I-V$ of the generator mesa, with marked bias points, is shown in the top panel of Fig. 2(a). From Fig. 1(d) it is seen that with increasing bias in the generator, critical currents in the detector are first reduced (red curve) and then get completely suppressed (blue curve)
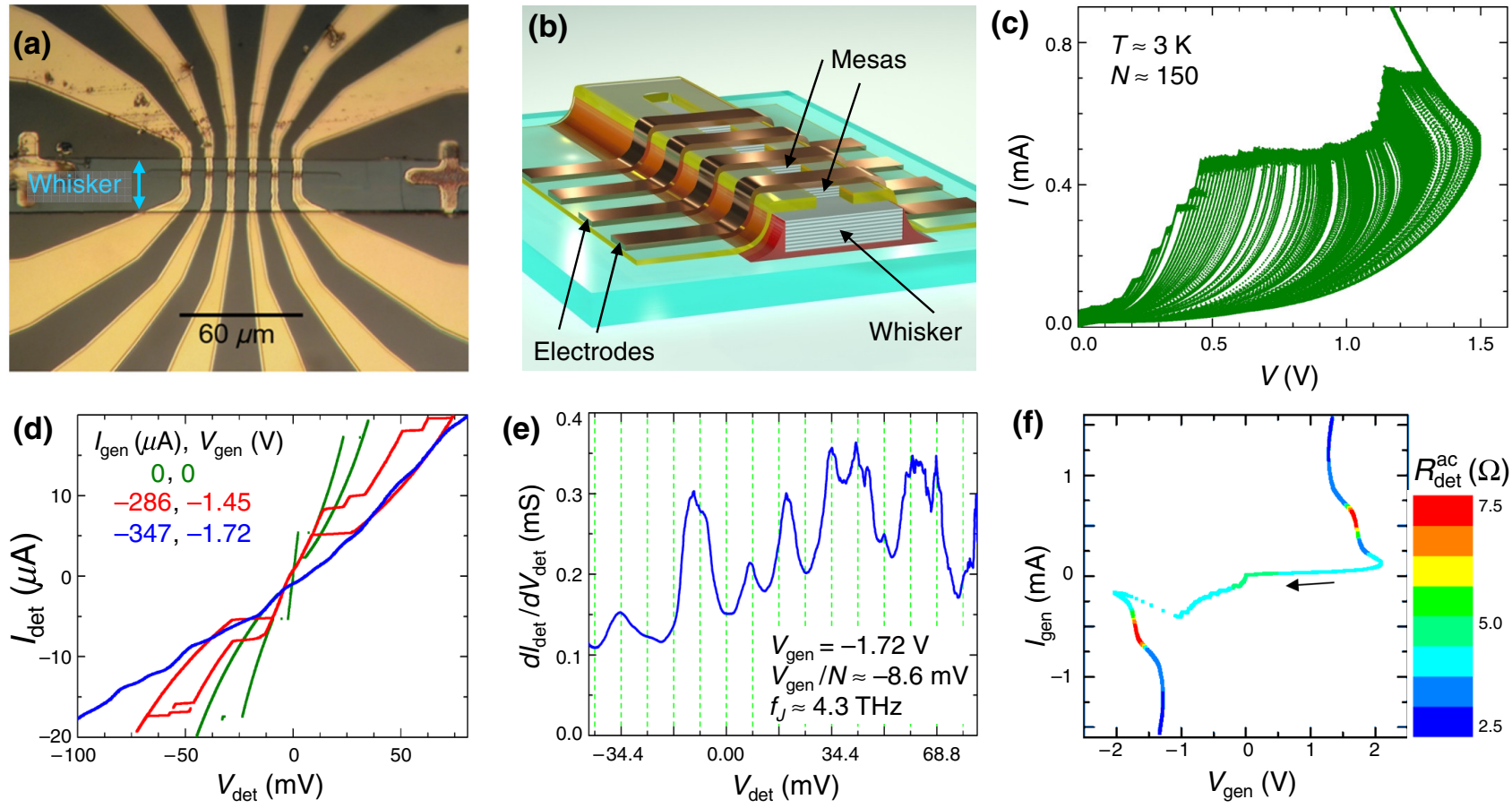

FIG. 1. (a) Optical image of one of the studied devices. Six mesas are formed beneath gold electrodes. (b) Sketch of the device. (c) $I-V$ characteristics (integrated oscillogram) of a mesa. Multiple branches correspond to switching of individual, $N \simeq 150, \mathrm{JJs}$ from the superconducting to the resistive state. (d) The $I-V$ curves of a detector mesa at three bias points in the generator, marked by circles in Fig. 2(a). (e) Differential conductance of the blue $I-V$ from (d). Peaks represent current steps. Grid lines correspond to expected Shapiro-step voltages. (f) On-chip generation-detection experiment with electrically disconnected mesas: Here we plot $I$ - $V$ of the generator mesa, color mapped by the ac resistance of electrically separated detector mesa on the same chip. The black arrow indicates the bias sweep direction. It is seen that the emission (red color) occurs at steps in the $I-V$. 

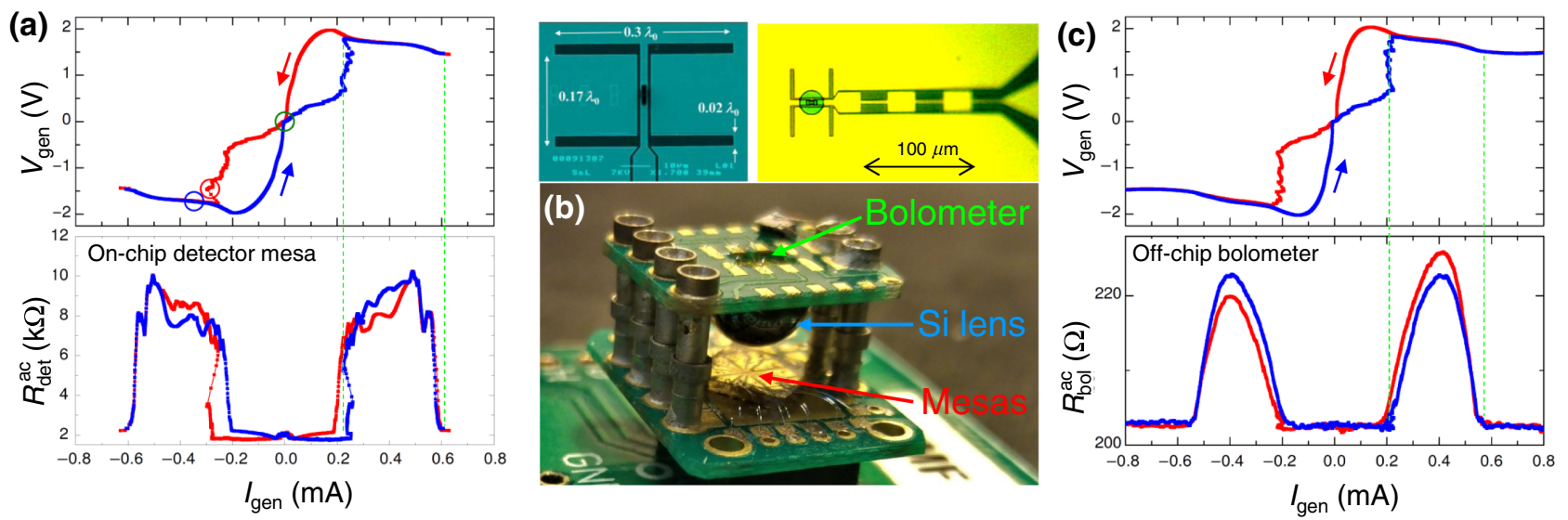

FIG. 2. Comparison of on-chip and off-chip detection schemes. (a) On-chip generation detection with electrically connected mesas. Top: the $V-I$ of a generator mesa at $T \simeq 3 \mathrm{~K}$. Circles represent bias points for the detector $I-V \mathrm{~s}$ in Fig. 1(d). Bottom: ac resistance of the detector mesa as a function of the generator current. Blue and red curves represent up and down bias sweeps. (b) A setup for offchip detection of emission by a NbN bolometer and images of the bolometer (from Refs. [38,39]). (c) Off-chip detection experiment. Top: the $V-I$ of the generator mesa. Bottom: the bolometer response as a function of $I_{\text {gen. }}$ Vertical dashed lines in (a),(c) indicate that emission occurs at the step in the generator $I-V$.

due to the EMW absorption [21]. Detailed dynamics of the generation-detection experiment is demonstrated in Video S1 within the Supplemental Material [35].

Absorption of EMW by a JJ leads to formation of Shapiro steps in the $I-V$ at $V_{n}=n h f / 2 e$ ( $n$ integer). Thus, junction response carries a spectroscopic information both about the frequency and the amplitude of EMW. In mesas with $N$ JJs, the EMW emission occurs at $f_{J}=2 e V_{\text {gen }} / N h$ (provided all JJs are synchronized). Thus, the primary Shapiro step should appear at $V_{\text {det }}=V_{\text {gen }} / N$. Small steps can indeed be seen in the blue $I-V$ from Fig. 1(d). Figure 1(e) shows the differential conductance for this curve. It exhibits clear peaks, corresponding to steps in the $I-V$. The grid spacing is equal to $V_{\text {gen }} / N$ with estimated $N \simeq 200$ for the generator mesa, see Fig. 2(a), and is in agreement with the observed peak separation. Some displacement of peak positions is likely caused by the fact that the detector response involves six JJs, see Fig. 1(d). A certain difference between JJs leads to a mismatch of bias conditions for appearance of Shapiro steps. This affects both the regularity and the amplitude of Shapiro steps. Nevertheless, the data is consistent with occurrence of monochromatic emission at $f_{J} \simeq 4.3 \mathrm{THz}$ because the nonmonochromatic emission would not lead to appearance of distinct steps.

To exclude possible electrical crosstalk, we perform a similar generation-detection experiment using electrically disconnected mesas. For this the whisker is cut by a focused ion beam in two sections, thus separating the generator and the detector mesas. The detector response remains qualitatively unchanged after such separation. Figure 1(f) shows the $I-V$ of the generator mesa, color maped by the response of electrically disconnected detector mesa (ac resistance, $R_{\text {det }}^{\mathrm{ac}}$, measured by the lockin technique with zero offset). It is seen that a profound, almost vertical step develops in the back-bending region of the $I-V$. The color code indicates that the detector response appears just at this step and disappears both above and below. In Fig. 2(a) the same behavior is demonstrated for the on-chip generation-detection experiment with electrically connected mesas. Here a correlation between the step in the $I-V$ of the generator and the upturn in the detector response is clearly seen (see also Video S1 within the Supplemental Material [35]). Such a nonmonotonic behavior precludes the self-heating origin of the observed signal and confirms occurrence of the EMW emission [21].

To confirm EMW emission into open space we perform off-chip detection using a $\mathrm{NbN}$ bolometer [38]. Figure 2(b) shows the measurement setup and images of the bolometer. The bolometer is placed at a distance approximately $1 \mathrm{~cm}$ above the device. Figure 2(c) shows corresponding generation (top) and detection (bottom) characteristics. Vertical dashed lines indicate that the bolometer response (lock-in resistance $R_{\mathrm{bol}}^{\mathrm{ac}}$ ) appears at the step in the $I_{\mathrm{gen}}-V_{\mathrm{gen}}$, thus confirming EMW emission. Some difference in the shapes of on-chip, Fig. 2(a), and off-chip, Fig. 2(c), responses is likely caused by the spectral selectivity of the doubleslot antenna of the bolometer, peaked at $f \simeq 1.6 \mathrm{THz}$, well below the emission frequency $f_{J} \simeq 4.2 \mathrm{THz}$ for this mesa [40].

Power dissipation leads to heating, but EMW emission-to radiative cooling of the device. We use this for unambiguous estimation of the emission power, $P_{\mathrm{THz}}$. Figure 3(a) represents the on-chip generation-detection measurement for another device (upward bias sweep). The sample stage of our dry cryostate has a relatively small heat 

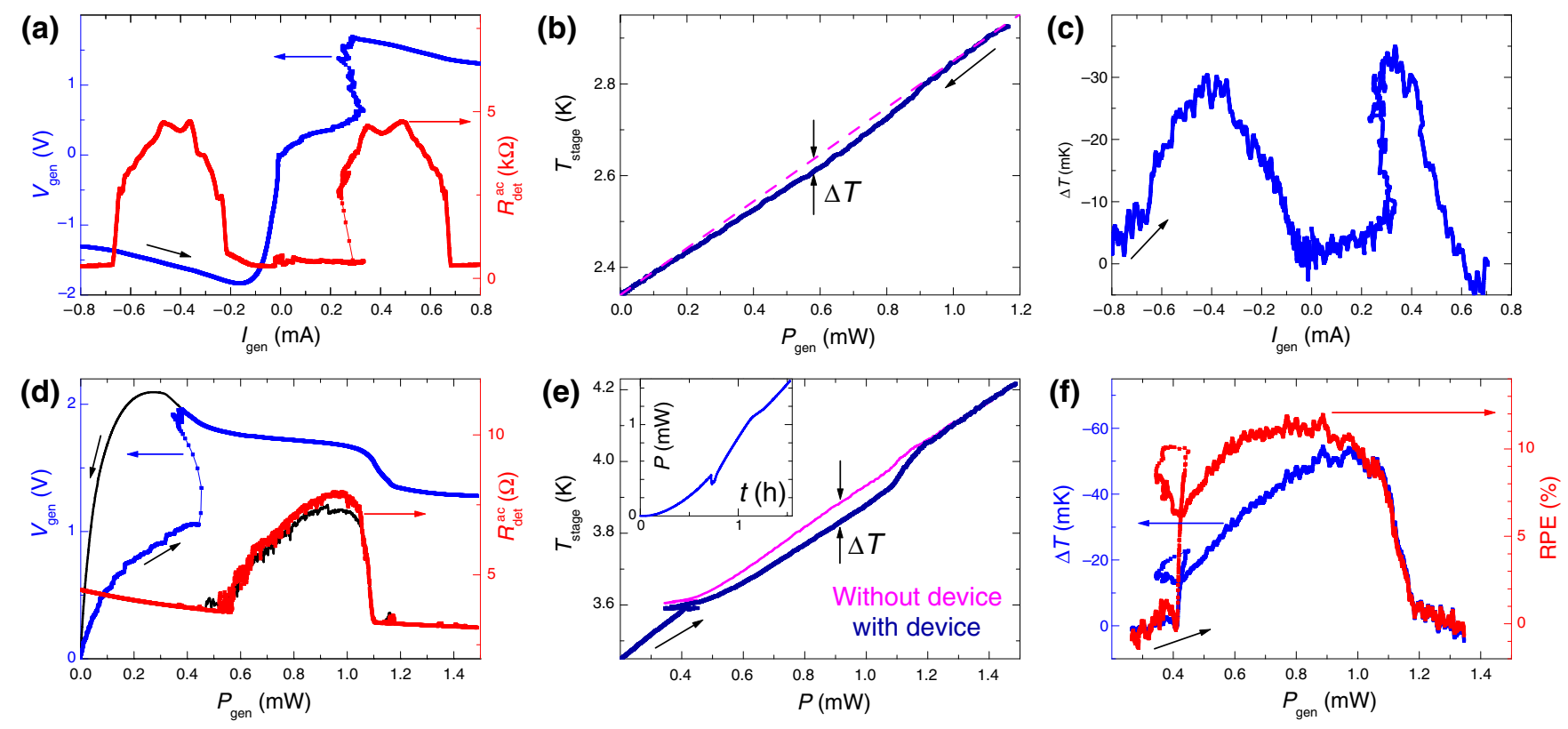

FIG. 3. The radiative cooling analysis for two devices (a)-(c) and (d)-(f). (a) The generator $V-I$ (blue, left axis) and the on-chip detector response (red, right axis) versus $I_{\text {gen }}$. (b) The stage temperature (dark blue) versus the dissipation power $P_{\text {gen }}$ for an upward bias sweep $\left(I_{\text {gen }}<0\right)$. It deviates from the linear dependence (magenta dashed line) at $P_{\text {gen }} \simeq 0.6 \mathrm{~mW}$. (c) The temperature drop versus $I_{\text {gen }}$. It is seen that $\Delta T$ is correlated with the detector response in (a). (d) The generator mesa voltage (blue, left axis) and the detector response (red, right axis) vs $P_{\text {gen }}$ for another device (an upward bias sweep). Black lines correspond to the reverse sweep. (e) $T_{\text {stage }}$ versus the dissipation power for experiments with (dark blue) and without (magenta) device for upward bias sweeps. The inset shows time dependencies of the dissipation power, identical for both cases. (f) Temperature drop (blue, left axis) and radiation power efficiency (red, right axis) versus $P_{\text {gen }}$. It is seen that RPE reaches $12 \%$.

capacitance and a finite heat resistance, $R_{\mathrm{th}}$, to the coldhead. Therefore, its temperature directly reflects the energy balance at the chip. Figure 3(b) shows the stage temperature as a function of the dissipation power, $P_{\text {gen }}=I_{\text {gen }} V_{\text {gen }}$, for the data from Fig. 3(a). As expected from Newton's law of cooling, it increases approximately linearly, $T_{\text {stage }} \simeq$ $T_{0}+R_{\mathrm{th}} P_{\text {gen }}$, with $R_{\mathrm{th}}=0.508 \mathrm{~K} / \mathrm{mW}$. However, at $P_{\text {gen }} \simeq$ $0.6 \mathrm{~mW}$ there is a visible drop down from the linear dependence. In Fig. 3 (c) we plot this drop, $\Delta T$, versus $I_{\text {gen }}$. It clearly follows the detector response, shown by the red line in Fig. 3(a). The correlation between $\Delta T$ and the EMW emission provides a straightforward demonstration of the radiative cooling phenomenon. It is worthwhile to note, it allows direct estimation of $P_{\mathrm{THz}}=\Delta T / R_{\mathrm{th}}$. The maximum drop $\Delta T=-31.5 \pm 2 \mathrm{mK}$ occurs at $I_{\text {gen }} \simeq 0.4 \mathrm{~mA}$ and $P_{\text {gen }}=0.6 \mathrm{~mW}$. This yields $P_{\mathrm{THz}} \simeq 62 \mu \mathrm{W}$ and RPE of $10.3 \pm 0.7 \%$ for this device.

Figures 3(d)-3(f) show similar measurements for another device. Figure 3(d) shows $V_{\text {gen }}$ (blue, left axis) and $R_{\text {det }}^{\text {ac }}$ (red, right axis) versus $P_{\text {gen }}$ for the upward bias sweep (black lines represent similar data for the reverse sweep). The dark blue curve in Fig. 3(e) shows $T_{\text {stage }}$ versus $P_{\text {gen }}$ for the upward sweep. Inset shows time dependence of $P_{\text {gen }}(t)$ during this sweep. It is nonlinear due to the nonOhmic $I-V$ of the generator. The nonlinearity may cause some transient effects. Therefore, to make an accurate calibration of the bare (without device) thermal response of the stage we heat it by a resistor, applying exactly the same time dependence of the dissipation power using a programmable current source. The corresponding variation of $T_{\text {stage }}$ is shown by the magenta line in Fig. 3(e). It is almost linear with the slope $R_{\mathrm{th}} \simeq 0.514 \mathrm{~K} / \mathrm{mW}$. Figure 3(f) shows the difference between $T_{\text {stage }}$ with and without device (blue) and the radiation power efficiency $\mathrm{RPE}=-\Delta T /\left(R_{\mathrm{th}} P_{\text {gen }}\right)$ (red curve). It is seen that the maximum RPE for this device reaches $12 \%$, which corresponds to the emission power of $P_{\mathrm{THz}} \simeq 0.11 \mathrm{~mW}$. We want to emphasize that this power is actually emitted in the far field. Indeed, it is taken out of the range cooled by the second stage of the cryocooler (which has a small cooling power) and is dumped into the first stage (with a very large cooling power), which is $10-40 \mathrm{~cm}$ away (see a sketch in Fig. S1 within the Supplemental Material [35]). This distance is much larger that the wavelength, $\lambda \sim 70 \mu \mathrm{m}$, at $f \sim 4.3 \mathrm{THz}$. Therefore, the radiative cooling probes the total far-field emission. Of course, for the practical device the emission should be collected and delivered via open space to the desired place, which inevitably involve certain losses. In this respect the estimation of RPE from the radiative cooling provides the upper limit of the achievable "useful" emission. 
Estimations above indicate that whisker-based $\mathrm{THz}$ sources can have RPE $>10 \%$, not far from the theoretical limit of $50 \%$ [34]. This significantly exceeds the RPE for devices made on regular Bi-2212 crystals [17,21]. Generally, the low RPE is caused by poor impedance matching with open space [34]. JJs are much smaller than the wavelength. Particularly tiny are interlayer distances of approximately $1 \mathrm{~nm}$. Therefore, JJs act as miniature dipoles with almost no emission in the far field. Essentially, the emission is facilitated by other passive but large-size elements of the device such as the Bi-2212 crystal and the electrodes, acting as matching antennas. Optimization of emission requires proper microwave design for impedance matching. In the Supplemental Material [35] we discuss the geometrical differences between crystal- and whiskerbased devices. We argue that the reported boost of RPE in whisker-based devices is cause by a specific turnstile antennalike geometry, which allows obviation of a large parasitic capacitance between the crystal and the electrode and facilitates good impedance matching with open space.

To conclude, a low-radiation power efficiency is one of the main problems of $\mathrm{THz}$ sources. For cryogenic sources the emission power is limited both by the cooling power and RPE: $P_{\mathrm{THz}}<\mathrm{RPE} \times P_{\text {cooling. }}$. For portable cryocoolers with limited $P_{\text {cooling, }}$, the only way to increase the emission power is by enhancement of the RPE. We present prototypes of $\mathrm{THz}$ sources based on $\mathrm{Bi}_{2} \mathrm{Sr}_{2} \mathrm{CaCu}_{2} \mathrm{O}_{8+\delta}$ whiskers with RPE up to $12 \%$, more than an order of magnitude larger than for similar devices made on regular Bi-2212 crystals. This indicates better impedance matching with open space due to the specific turnstile antennalike geometry of whisker-based devices. We conclude that such devices can be used for creation of tunable, monochromatic, cw, compact and, what is more, power-efficient $\mathrm{THz}$ sources.

Acknowledgments. The work is supported by the Russian Science Foundation Grant No. 19-19-00594. We are grateful to A. Agostino and M. Truccato for assistance with whisker preparation, to S. Cherednichenko for providing the bolometer and to A. Efimov and K. Shiianov for assistance in experiment. The paper was written during a sabbatical semester of V.M.K. at MIPT.

[1] M. Tonouchi, Cutting-edge terahertz technology, Nat. Photonics 1, 97 (2007).

[2] There are also pulsed broad-band $\mathrm{THz}$ sources, that are fairly well developed today, for a review see, e.g., P. Salen et al., Matter manipulation with extreme terahertz light: progress in the enabling THz technology. Phys. Rep. 836837, 1-74 (2019). Such sources are beyond the scope of our paper, which is focusing on monochromatic cw sources.
[3] The dissipation power is $P=I V$, where $I$ and $V$ are bias current and voltage at the operation point of the device. Other definitions are also used, such as the wall plug efficiency, which also takes into account, e.g., the power required for refrigeration of the device. However, we use our RPE definition because operation of the cryogenic device is limited primarily by self-heating and, therefore, its performance depends solely on the power dissipated inside the cryostat.

[4] M. Razeghi, Q. Y. Lu, N. Bandyopadhyay, W. Zhou, D. Heydari, Y. Bai, and S. Slivken, Quantum cascade lasers: From tool to product, Opt. Express 23, 229245 (2015).

[5] M. A. Belkin and F. Capasso, New frontiers in quantum cascade lasers: High performance room temperature terahertz sources, Phys. Scripta 90, 118002 (2015).

[6] A. Khalatpour, A. K. Paulsen, C. Deimert, Z. R. Wasilewski, and Q. Hu, High-power portable terahertz laser systems, Nat. Photonics 15, 16 (2021).

[7] A. Lyakh, M. Suttinger, R. Go, P. Figueiredo, and A. Todi, $5.6 \mu \mathrm{m}$ quantum cascade lasers based on a two-material active region composition with a room temperature wallplug efficiency exceeding 28\%, Appl. Phys. Lett. 109, 121109 (2016).

[8] X. Wang, C. Shen, T. Jiang, Z. Zhan, Q. Deng, W. Li, W. $\mathrm{Wu}, \mathrm{N}$. Yang, W. Chu, and S. Duan, High-power terahertz quantum cascade lasers with $\sim 0.23 \mathrm{~W}$ in continuous wave mode, AIP Adv. 6, 075210 (2016).

[9] C. A. Curwen, J. L. Reno, and B. S. Williams, Broadband continuous single-mode tuning of a short-cavity quantumcascade VECSEL, Nat. Photonics 13, 855 (2019).

[10] C. Walther, M. Fischer, G. Scalari, R. Terazzi, N. Hoyler, and J. Faist, Quantum cascade lasers operating from 1.2 to 1.6 THz, Appl. Phys. Lett. 91, 131122 (2007).

[11] K. Vijayraghavan, Y. Jiang, M. Jang, A. Jiang, K. Choutagunta, A. Vizbaras, F. Demmerle, G. Boehm, M. C. Amann, and M. A. Belkin, Broadly tunable terahertz generation in mid-infrared quantum cascade lasers, Nat. Commun. 4, 2021 (2013).

[12] M. Rösch, G. Scalari, M. Beck, and J. Faist, Octavespanning semiconductor laser, Nat. Photonics 9, 42 (2015).

[13] S. Han, B. Bi, W. Zhang, and J. E. Lukens, Demonstration of Josephson effect submillimeter wave sources with increased power, Appl. Phys. Lett. 64, 1424 (1994).

[14] P. Barbara, A. B. Cawthorne, S. V. Shitov, and C. J. Lobb, Stimulated Emission and Amplification in Josephson Junction Arrays, Phys. Rev. Lett. 82, 1963 (1999).

[15] V. P. Koshelets and S. V. Shitov, Integrated superconducting receivers, Supercond. Sci. Techn. 13, R53 (2000).

[16] L. Ozyuzer, A. E. Koshelev, C. Kurter, N. Gopalsami, Q. Li, M. Tachiki, K. Kadowaki, T. Yamamoto, H. Minami, H. Yamaguchi, T. Tachiki, K. E. Gray, W.-K. Kwok, and U. Welp, Emission of coherent $\mathrm{THz}$ radiation from superconductors, Science 318, 1291 (2007).

[17] T. M. Benseman, K. E. Gray, A. E. Koshelev, W.-K. Kwok, U. Welp, H. Minami, K. Kadowaki, and T. Yamamoto, Powerful terahertz emission from $\mathrm{Bi}_{2} \mathrm{Sr}_{2} \mathrm{CaCu}_{2} \mathrm{O}_{8+\delta}$ mesa arrays, Appl. Phys. Lett. 103, 022602 (2013).

[18] U. Welp, K. Kadowaki, and R. Kleiner, Superconducting emitters of THz radiation, Nat. Photonics 7, 702 (2013).

[19] T. Kashiwagi et al., Efficient Fabrication of IntrinsicJosephson-Junction Terahertz Oscillators with Greatly 
Reduced Self-Heating Effects, Phys. Rev. Appl. 4, 054018 (2015).

[20] X. Zhou, Q. Zhu, M. Ji, D. An, L. Hao, H. Sun, S. Ishida, F. Rudau, R. Wieland, J. Li, D. Koelle, H. Eisaki, Y. Yoshida, T. Hatano, R. Kleiner, H. Wang, and P. Wu, Three-terminal stand-alone superconducting terahertz emitter, Appl. Phys. Lett. 107, 122602 (2015).

[21] E. A. Borodianskyi and V. M. Krasnov, Josephson emission with frequency span 1-11 $\mathrm{THz}$ from small $\mathrm{Bi}_{2} \mathrm{Sr}_{2} \mathrm{CaCu}_{2} \mathrm{O}_{8+\delta}$ mesa structures, Nat. Commun. 8, 1742 (2017).

[22] M. A. Galin, E. A. Borodianskyi, V. V. Kurin, I. A. Shereshevskiy, N. K. Vdovicheva, V. M. Krasnov, and A. M. Klushin, Synchronization of Large Josephson-Junction Arrays by Traveling Electromagnetic Waves, Phys. Rev. Appl. 9, 054032 (2018).

[23] H. Sun et al., Compact High- $T_{c}$ Superconducting Terahertz Emitter Operating up to 86 K, Phys. Rev. Appl. 10, 024041 (2018).

[24] T. Kashiwagi, T. Yuasa, Y. Tanabe, T. Imai, G. Kuwano, R. Ota, K. Nakamura, Y. Ono, Y. Kaneko, M. Tsujimoto, H. Minami, T. Yamamoto, R. A. Klemm, and K. Kadowaki, Improved excitation mode selectivity of high- superconducting terahertz emitters, J. Appl. Phys. 124, 033901 (2018).

[25] H. Zhang et al., Resonant Cavity Modes in $\mathrm{Bi}_{2} \mathrm{Sr}_{2} \mathrm{CaCu}_{2}$ $\mathrm{O}_{8+x}$ Intrinsic Josephson Junction Stacks, Phys. Rev. Appl. 11, 044004 (2019).

[26] G. Kuwano, M. Tsujimoto, Y. Kaneko, T. Imai, Y. Ono, S. Nakagawa, S. Kusunose, H. Minami, T. Kashiwagi, K. Kadowaki, Y. Simsek, U. Welp, and W.-K. Kwok, MesaSidewall Effect on Coherent Terahertz Radiation via Spontaneous Synchronization of Intrinsic Josephson Junctions in $\mathrm{Bi}_{2} \mathrm{Sr}_{2} \mathrm{CaCu}_{2} \mathrm{O}_{8+\delta}$, Phys. Rev. Appl. 13, 014035 (2020).

[27] M. Tsujimoto, S. Fujita, G. Kuwano, K. Maeda, A. Elarabi, J. Hawecker, J. Tignon, J. Mangeney, S. S. Dhillon, and I. Kakeya, Mutually Synchronized Macroscopic Josephson Oscillations Demonstrated by Polarization Analysis of Superconducting Terahertz Emitters, Phys. Rev. Appl. 13, 051001(R) (2020).

[28] Y. Saiwai, T. Kashiwagi, K. Nakade, M. Tsujimoto, H. Minami, R. A. Klemm, and K. Kadowaki, Liquid heliumfree high- $T_{c}$ superconducting terahertz emission system and its applications, Jpn. J. Appl. Phys. 59, 105004 (2020).

[29] K. Delfanazari, R. A. Klemm, H. J. Joyce, D. A. Ritchie, and K. Kadowaki, Integrated, portable, tunable, and coherent terahertz sources and sensitive detectors based on layered superconductors, Proc. IEEE 108, 721 (2020).

[30] M. A. Galin, F. Rudau, E. A. Borodianskyi, V. V. Kurin, D. Koelle, R. Kleiner, V. M. Krasnov, and A. M. Klushin, Direct Visualization of Phase-Locking of Large Josephson Junction Arrays by Surface Electromagnetic Waves, Phys. Rev. Appl. 14, 024051 (2020).

[31] V. M. Krasnov, A. Yurgens, D. Winkler, P. Delsing, and T. Claeson, Evidence for Coexistence of the Superconducting Gap and the Pseudogap in Bi-2212 from Intrinsic Tunneling Spectroscopy, Phys. Rev. Lett. 84, 5860 (2000).

[32] V. M. Krasnov, Temperature dependence of the bulk energy gap in underdoped $\mathrm{Bi}_{2} \mathrm{Sr}_{2} \mathrm{CaCu}_{2} \mathrm{O}_{8+\delta}$ : Evidence for the mean-field superconducting transition, Phys. Rev. B 79, 214510 (2009).

[33] R. Kleiner and P. Müller, Intrinsic Josephson effects in high$T_{c}$ superconductors, Phys. Rev. B 49, 1327 (1994).

[34] V. M. Krasnov, Coherent flux-flow emission from stacked Josephson junctions: Nonlocal radiative boundary conditions and the role of geometrical resonances, Phys. Rev. B 82, 134524 (2010).

[35] See Supplemental Material at http://link.aps.org/supplem ental/10.1103/PhysRevApplied.16.L061001 for additional information about the following: (a) Sample fabrication; (b) experimental setup; (c) switching current detection in onchip generation-detection experiment; (d) video S1 of onchip generation detection; and (e) discussion of geometrical advantages of whisker devices.

[36] V. M. Krasnov, A. Yurgens, and P. Delsing, Self-heating in small mesa structures, J. Appl. Phys. 89, 5578 (2001).

[37] V. M. Krasnov, M. Sandberg, and I. Zogaj, In Situ Measurement of Self-Heating in Intrinsic Tunneling Spectroscopy, Phys. Rev. Lett. 94, 077003 (2005).

[38] S. Cherednichenko, V. Drakinskiy, T. Berg, P. Khosropanah, and E. Kollberg, Hot-electron bolometer terahertz mixers for the herschel space observatory, Rev. Sc. Instr. 79, 034501 (2008).

[39] H. Motzkau, PhD Thesis, Stockholm University 2015, ISBN 978-91-7649-157-7.

[40] The spectral selectivity is determined solely by the antenna. The bolometer itself has a much broader frequency range and can operate at such a frequency, as demonstrated by P. Khosropanah, J.R. Gao, W.M. Laauwen, M. Hajenius, and T.M. Klapwijk, Low noise $\mathrm{NbN}$ hot electron bolometer mixer at 4.3 THz. Appl. Phys. Lett. 91, 221111 (2007). 